\title{
Study of Serum TSH in Healthy Individuals on Non-Vegetarian and Lacto-Vegetarian Diet
}

\author{
Nazim Saifi ${ }^{1}$, Nazmeen ${ }^{2}$, Yusuf Jamal ${ }^{3}$ \\ ${ }^{1}$ Lecturer (Physiology), SUMC, East Champaran, Bihar \\ ${ }^{2}$ Associate Professor (Physiology), A \& U Tibbia College, Karol Bagh \\ ${ }^{3}$ Professor (Physiology), A \& U Tibbia College, Karol Bagh
}

\begin{abstract}
Nutrition plays a critical role in thyroid hormone synthesis. The production of thyroid hormones depends upon adequate amount of tyrosine, a non essential amino acid synthesized in the body from the essential amino acid phenylalanine, and dietary iodine. Moreover selenium, zinc and iron also help in thyroid hormone synthesis and in their action on target cells. There is perception in general that vegetarian diet is healthy and balanced diet but it is also fact that the vegetarian diet is deficient of many major nutrients along with iodine. Iodine content in food of plant origin (vegetarian diet) is lower in comparison with that of animal origin due to a low iodine concentration in soil. In areas of relative iodine deficiency, there is an increased prevalence of goiter and hypothyroidism in India. The present study was conducted in Karol Bagh area of Delhi, on non-vegetarian and lacto-vegetarian diet healthy individuals; their serum TSH range was assessed, as TSH is the best indicator of thyroid functions. The study showed that the TSH range is insignificantly lower in lacto-vegetarian people, which is not in accordance with previous studies. This study reflects that lactovegetarian diet is not associated with hypothyroidism.
\end{abstract}

Keywords: thyroid stimulating hormones, iodine deficiency, lacto-vegetarian diet

\section{Introduction}

The main regulator of thyroid function is thyroid-stimulating hormone (TSH), a glycoprotein synthesized and secreted from the thyrotrope cells of the anterior pituitary gland, which in turn is under the control of TRH secreted by the hypothalamus, (Braverman and Cooper, 2013). Within the thyroid gland, TSH (Thyroid Stimulating Hormone) stimulates virtually all metabolic and cellular processes involved in the synthesis and secretion of thyroid hormones, including iodine uptake and organification, thyroglobulin synthesis, iodotyrosine coupling . Clinically observable effects of TSH on the normal thyroid gland include thyroid gland enlargement, increased radioactive iodine uptake, and increased secretion of thyroxine (T4) and T3 (Becker, 2001). The formation and secretion of thyroid hormones are regulated by Thyroid Stimulating Hormone (TSH) through negative feedback system (Fauci et. al., 2008). Thyroid hormones play a very critical role in cell differentiation during development and help maintain thermogenic and metabolic homeostasis in the adult. Thyroid hormones enhance the rate of oxygen consumption, utilization of fats, carbohydrates and proteins by the cells. In this respect, the thyroid gland undertakes a managerial role in the regulation of metabolic functions. Nutrition plays a critical role in thyroid hormone synthesis. The production of thyroid hormones depends upon adequate amount of tyrosine, a non essential amino acid synthesized in the body from the essential amino acid phenylalanine, and dietary iodine. (Whitney et. al. 2005) Moreover, other than iodine, selenium, zinc and iron also help in thyroid hormone synthesis and in their action on target cells. T4 is the principle hormone secreted by the thyroid gland but has little biological activity. T3 the major biological active hormone is also produced by the thyroid gland, but most of this metabolite is derived from the de-iodination of T4. De- iodinase enzyme D1 is located primarily in liver and kidney and the enzymatic activity is low in hypothyroidism and high in hyperthyroidism (Sing-Yung \& Visser, 1994). Deiodinase enzyme D1 is a selenoprotein, which is formed by the addition of selenium to the amino acid selenocystein. Selenium deficient diets have been studied and have high incidents of goiter and reduced serum T3 concentration. (Melmed \& Conn, 2005) Iron deficiency impairs the synthesis of thyroid hormones by reducing the activity of heme-dependent thyroid peroxidase. Population studies have revealed that nonanemic children responded swiftly to iodine supplementation with regard to goiter and TSH levels than anemic children. (Zimmermann \& Kohrle, 2002) Zinc status also affects thyroid function. Zinc is required for the proper functioning of D1 de-iodinase enzyme, the enzyme required for the conversion of thyroxine into tri-idothyronine (De Groot \& Jameson, 2010)

Individual who eat no animal flesh nor any food of animal origin. They are called strict vegetarian or vegan. Individual who eat plant proteins, and also use milk are called lactovegetarian. Lacto-ova vegetarians are those individuals who eat both milk and eggs along with plant proteins. (Stanfield \& Hui, 2010) In India vegetarian people usually do not eat egg but consume milk and milk product, so they can be termed as lacto vegetarian. There is increasing perception among people that vegetarian diet is balanced and healthy diet and prevents from various chronic diseases. But it is also fact that strict vegetarian (vegan) diet is deficient of many major nutrients like calcium, vitamin D3, zinc and iodine. (Stanfield \& Hui, 2010) Iodine content in food of plant origin (vegetarian diet) is lower in comparison with that of animal origin due to a low iodine concentration in soil. (Krajcovicová et. al. 2003) Iodine deficiency is prevalent in many mountainous regions and in central Africa, central South America, and northern Asia. In areas of relative iodine deficiency, there is an increased prevalence 


\section{International Journal of Science and Research (IJSR) \\ ISSN (Online): 2319-7064}

Index Copernicus Value (2015): 78.96 | Impact Factor (2015): 6.391

of goiter and, when deficiency is severe, hypothyroidism and cretinism. (Fauci et. al., 2008)Iodine deficiency is a problem in India, the importance of iodine deficiency cannot be underestimated in the Indian context. (Ambika et.al. 2011) Researchers from New Delhi had shown that this was linked to iodine deficiency and that this resulted in decompensated hypothyroidism in many cases. The purpose of this study was to assess the serum TSH range difference among nonvegetarian and lacto-vegetarian young healthy individuals.

\section{Method}

The lacto-vegetarian and non vegetarian, one hundred (100) young healthy volunteers were randomly selected. The aim of this research is, to study the possible relation between serum TSH and lacto-vegetarian and non vegetarian diets. It was a cross sectional, descriptive study and was carried out in the department of Physiology of A \& U Tibbia College, Karol Bagh, Delhi, during the period extended from 2014 to 2015.

\subsection{Inclusion Criteria}

- Individuals of 18 to 25 years of age

- Either sex

\subsection{Exclusion Criteria}

- Known cases of Hyperthyroidism or Hypothyroidism

- Pregnant women

- Individual with history of alcohol intake/tobacco chewing/smoking

\subsection{Determination of Thyroid Stimulating Hormone (TSH)}

\section{Blood Sample Collection}

Individuals were called empty stomach in the morning for blood sample collection. $3 \mathrm{ml}$ blood was drawn from median cubital vein by sterile, disposable $5 \mathrm{ml}$ syringes. After that blood was collected in a plain vial and allowed to clot at room temperature for an hour and then serum was obtained after centrifugation for 5 minutes at $3000 \mathrm{rpm}$ in centrifugal machine.

\section{Test Kits}

Serological test of thyroid stimulating hormone (TSH) was done by URIT-660 Auto Analyzer and the testing kit of MERIL Company was used.

\section{Observation}

Total 100 volunteers were randomly selected for Mizaj identification and Thyroid function test as per inclusion criteria, out of which 6 individuals had higher TSH value, above normal limit, so those volunteers were excluded from statistical analysis of the study. Finally total 94 volunteers were completed the study.

Diet Wise Distribution
\begin{tabular}{|c|c|c|}
\hline Diet & No. of Subjects & Percentage $\%$ \\
\hline Lacto-Vegetarian & 22 & $23 \%$ \\
\hline Non-Vegetarian & 72 & $77 \%$ \\
\hline
\end{tabular}

In all 94 volunteers $23 \%$ were vegetarian and $77 \%$ were mix or non-vegetarian. Total 94 patients were divided into two groups' i.e. non-vegetarian diet group and lacto-vegetarian diet group and in both the groups mean of TSH was calculated.

\section{Mean \pm Sd of TSH, According To Diet}

\begin{tabular}{|c|c|}
\hline Diet & TSH $($ Mean \pm Sd $)$ \\
\hline Non-Vegetarian & $2.31 \pm 1.39$ \\
\hline Lacto-Vegetarian & $2.55 \pm 1.07$ \\
\hline
\end{tabular}

Mean of TSH value in vegetarian diet $(2.31 \pm 1.39)$ is higher than mix diet $(2.55 \pm 1.07)$

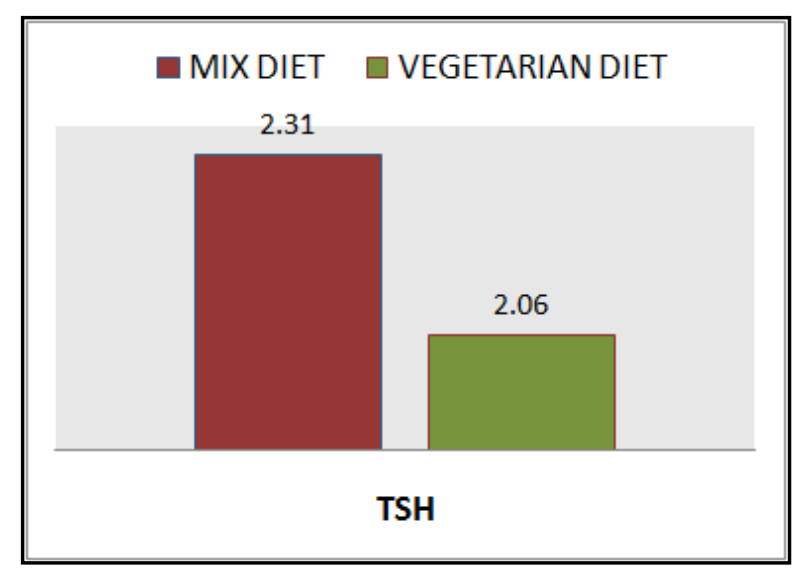

T-test is applied to check the statistical significance in TSH mean of lacto-vegetarian and non-vegetarian diet groups. The results are as follows $\mathrm{t}=0.7442, \mathrm{df}=92$, standard error of difference $=0.322$ and $\mathrm{p}=0.4586$, which shows the result is insignificant. $(p<0.05)$

\section{Conclusion}

Out of 94 volunteers 22 (23\%) were lacto-vegetarian and 72 (77\%) were non-vegetarian (Table-7). Total 94 individuals were divided into two groups i.e. non-vegetarian diet group and lacto-vegetarian diet group and in both the groups mean of TSH was calculated (Table-8). Mean of TSH value in non-vegetarian $(2.31 \pm 1.39)$ is lower than lacto-vegetarian diet (2.55 \pm 1.07$)$. Lacto-vegetarian diet is comparatively iodine deficient diet and such people are considered having increased risk of hypothyroidism, therefore it may be assumed that lacto vegetarian's diet people may have TSH level relatively at higher side and FT3, FT4 at lower side, within the normal range, in comparison to non-vegetarian. This study showed lacto-vegetarian has insignificant high level of TSH in comparison to non-vegetarian diet, which reflects that lacto-vegetarian people of Delhi are not iodine deficient, as they consume salt which is fortified with iodine.

\begin{tabular}{|c|c|c|}
\hline Total Registered Volunteers & Excluded & Completed \\
\hline 100 & 6 & 94 \\
\hline
\end{tabular}




\section{Result}

Based on the various observations it is found that TSH mean, within the normal range, is slightly higher in Lactovegetarian people in comparison to Non-vegetarian, but is not statistically significant. This study shows that there is no correlation between Lacto-vegetarian diet and TSH, which reflects that lacto-vegetarian people of Karol Bagh area, Delhi are not iodine deficient; the reason may be as they consume salt which is fortified with iodine. The present study was restricted to only 100 volunteers. Thus, it may not be comprehensive. Therefore, future researches may be conducted on much larger sample. The results of such studies may provide greater insight for the determination of prevalence of hypothyroidism and goiter in the society.

\section{References}

[1] Stanfield P S, Hui Y H. 2010. Nutrition and Diet Therapy: Self-Instructional Approaches, $5^{\text {th }}$ ed. Jones and Bartlett publishers, Ontario, Canada, p40

[2] Ambika, Gopalakrishnan, Unnikrishnan and Menon U $\mathrm{V}$, Thyroid disorders in India: An epidemiological perspective, Indian J Endocrinol Metab. 2011 Jul; 15(Supp12): S78-S81

[3] Braverman, L. E., Cooper, D. S. 2013. Werner \& Ingbar's The Thyroid A Fundamental And Clinical Text, 10th Ed. Lippincott Williams \& Wilkins, Philadelphia, p612

[4] Fauci A S, Braunwald E, Kasper D L, Hauser S L, Longo D L, Jameson J L, Loscalzo J. 2008. Harrison's Principles of Internal Medicine, $17^{\text {th }}$ Ed. Mc Graw Hill New York. Pp2224

[5] Whitney, Eleanor and Sharon Rolfes. 2005. Understanding Nutrition. $9^{\text {th }} \quad$ Ed. Belmont, CA:Thompson-Wadsworth, pp183

[6] Sing-Yung Wu, Visser T J. 1994. Thyroid Hormone Metabolism-Molecular Biology and Alternate Pathways, CRC Press, Boca Raton Florida, pp 67

[7] Melmed S, Conn P M. 2005. Endocrinology: Basic and Clinical Principles, $2^{\text {nd }}$ Ed. Humana Press, Totowa, New Jersey, pp 273

[8] Zimmermann MB, Köhrle J. 2002. The impact of iron and selenium deficiencies on iodine and thyroid metabolism: Biochemistry and relevance to public health. Thyroid 2002;12:867-78

[9] De Groot L J, Jameson J L. 2010. Endocrinology Adult and Pediatric: The Thyroid Gland E-Book, Elsevier saunders, Philedelphia, pp e314

[10] Krajcovicová-Kudlácková $\quad M^{1}$, Bucková $\quad \mathrm{K}$, Klimes I, Seboková E. Iodine deficiency in vegetarians and vegans. Ann Nutr Metab. 2003;47(5):183-5

[11] Becker K L, 2001. Principles and Practice of Endocrinology and Metabolism. $3^{\text {rd }}$ Ed. Lippincott Williams and wilkins, Philadelphia, USA, pp957 